\title{
Advantages of a Modular Mars Surface Habitat Approach
}

\author{
Michelle A. Rucker \\ NASA Johnson Space Center \\ 2101 NASA Parkway \\ Houston, TX 77058 \\ 281-244-5569 \\ michelle.a.rucker@nasa.gov \\ Alida Andrews \\ The Aerospace Corporation \\ 2525 Bay Area Blvd \\ Houston, TX 77058 \\ 281-244-7902 \\ alida.andrews-1@nasa.gov
}

\author{
Stephan J. Hoffman, Ph.D. \\ The Aerospace Corporation \\ 2525 Bay Area Blvd \\ Houston, TX 77058 \\ 281-483-9264 \\ stephen.j.hoffman@nasa.gov \\ Kevin Watts \\ NASA Johnson Space Center \\ 2101 NASA Parkway \\ Houston, TX 77058 \\ 281-244-8265 \\ kevin.d.watts@nasa.gov
}

Keywords: Mars, Surface, Human, Habitat

\begin{abstract}
Early crewed Mars mission concepts developed by the National Aeronautics and Space Administration (NASA) assumed a single, large habitat would house six crew members for a 500-day Mars surface stay. At the end of the first mission, all surface equipment - including the habitat--would be abandoned and the process would be repeated at a different Martian landing site. This work was documented in a series of NASA publications culminating with the Mars Design Reference Mission 5.0 (NASA-SP-2009-566). The Evolvable Mars Campaign (EMC) explored whether re-using surface equipment at a single landing site could be more affordable than the Apollo-style explore-abandon-repeat mission cadence. Initial EMC assumptions preserved the single, monolithic habitat - the only difference being a new requirement to reuse the surface habitat for multiple expedition crews. A trade study comparing a single large habitat versus smaller, modular habitats leaned towards the monolithic approach as more mass-efficient. More recent work has focused on the operational aspects of building up Mars surface infrastructure over multiple missions, and has identified compelling advantages of the modular approach that should be considered before making a final decision. This paper explores Mars surface mission operational concepts and integrated system analysis, and presents an argument for the modular habitat approach.
\end{abstract}

